Bio - grafia. Escritos sobre la Biología y su Enseñanza. ISSN 2027

Edición Extraordinaria. p.p. $905-916$

Memorias del VIII Encuentro Nacional de Experiencias en Enseñanza de la Biología y la Educación Ambiental. III Congreso Nacional de Investigación en Enseñanza de la Biología.

\title{
REFERENTE EPISTEMOLÓGICO EN EL ÉNFASIS DE INVESTIGACIÓN SOBRE EL CONOCIMIENTO PROFESIONAL DEL PROFESOR, A PROPÓSITO DE LA DIDÁCTICA COMO DISCIPLINA
}

\section{EPISTEMOLOGICAL REFERENCE IN THE RESEARCH PROMINENCE ABOUT THE TEACHER'S PROFESSIONAL KNOWLEDGE, PURPOSELY OF DIDACTIC AS DISCIPLINE}

Enciso Orjuela Marisol'; Roa Acosta Robinson ${ }^{2}$

"Cuando un cuerpo se encuentra con otro cuerpo distinto o una idea con otra distinta, sucede o bien que sus relaciones se componen formando un todo más poderoso, o bien que una de éstas descompone a la otra y destruye la cohesión de sus partes." Gilles Deleuze

\section{Resumen}

Las diferentes tendencias de investigación del conocimiento profesional del profesor se vinculan con diversos referentes epistemológicos que dan cuenta de la naturaleza, las condiciones de validez y las posibilidades de conocimiento que emergen en y para la profesión docente, reconociendo la existencia de un conocimiento didáctico especializado que se legitima en su ejercicio profesional y en la vinculación con una comunidad académica, cuyo territorio toma distancia epistemológica de las "disciplinas puras y duras" para consolidarse en la disciplina de la didáctica. En este orden, la didáctica se posiciona como una disciplina que emerge en unas condiciones históricas particulares y que se ha venido consolidando en una comunidad académica con unos énfasis de investigación que aportan a la disciplina misma. Reflexionar y construir desde la didáctica, es asumir un potente

\footnotetext{
${ }^{1}$ Licenciada en Educación Básica de la Universidad Pedagógica y tecnológica de Tunja (UPTC). Estudiante de maestría en Educación de la Universidad Pedagógica Nacional. Correo: karenasol13@gmail.com

2 Estudiante de Doctorado en Educación, Universidad Pedagógica Nacional. Magíster en Educación, Universidad Externado de Colombia. Licenciado en Biología. Universidad Pedagógica Nacional. Profesor de la Secretaria de Educación de Bogotá y profesor de cátedra Universidad Pedagógica Nacional. Bogotá. Colombia.robinsonroa@hotmail.com
} 
Bio - grafia. Escritos sobre la Biología y su Enseñanza. ISSN 2027

Edición Extraordinaria. p.p. $905-916$

Memorias del VIII Encuentro Nacional de Experiencias en Enseñanza de la Biología y la Educación Ambiental. III Congreso Nacional de Investigación en Enseñanza de la Biología.

referente epistemológico que brinda elementos analíticos, discursivos y metodológicos en la reflexión sistemática del conocimiento del profesor.

Palabras claves:

Referente epistemológico, disciplina didáctica, conocimiento del profesor.

\section{Abstract}

The different research tendencies about the teacher's professional knowledge are linked with various epistemological references to realize the nature, the conditions of validity and the possibilities of knowledge that emerges in the teaching profession, recognizes the existence of a specific knowledge that legitimizes his practice and connection with an academic community that takes epistemological distance from the "pure and core disciplines" in order to consolidate in the discipline of didactic. Thus, didactics is positioned as an emerging discipline in particular historical conditions and it is being consolidated in an academic community with such research focused on obtaining contributions to its own discipline. Reflect and build from didactics, it is to assume a powerful epistemological reference that provide analytical, discursive and methodological elements in the systematic reflection of teachers' knowledge.

\section{Keywords:}

Epistemological reference, discipline of didactics, teacher's knowledge.

\section{Introducción}

Las diferentes tendencias de investigación en el énfasis ${ }^{3}$ del conocimiento profesional del profesor -este, entre otros énfasis en el campo de investigación de la didáctica de la ciencias- se vinculan con diversas posturas epistemológicas- ontológicas y metodológicas, en un intento por explicar la naturaleza, los criterios de validación y las distintas maneras como se configura el conocimiento del profesor. En este sentido, se posiciona el

\footnotetext{
${ }^{3}$ Se entiende en este escrito que la didáctica como disciplina está contenida por varios énfasis de investigación, uno de ellos es del conocimiento del profesor, el cual a la vez contiene varias tendencias de investigación, como se mostrará más adelante. No se hace alusión a la palabra línea de investigación, pues el conocimiento no responde a una construcción lineal que emerge en un solo punto de manera unidimensional y unidireccional. Por el contrario, se trata de asumir dicha concepción desde la apertura a la diversidad de posibilidades con múltiples dimensiones y diversos enfoques.
} 
Bio - grafia. Escritos sobre la Biología y su Enseñanza. ISSN 2027

Edición Extraordinaria. p.p. 905- 916

Memorias del VIII Encuentro Nacional de Experiencias en Enseñanza de la Biología y la Educación Ambiental. III Congreso Nacional de Investigación en Enseñanza de la Biología.

"conocimiento didáctico" como uno de los referentes epistemológicos que dan sustrato a los procesos de investigación vinculados con el énfasis sobre el conocimiento profesional del profesor, en tanto que implican un "conocimiento" que se ha venido reconfigurando y aportando a las disciplinas de la pedagogía y la didáctica, las cuales son resultado de una trayectoria histórica particular, el reconocimiento de un status social a la profesión docente, así como unas condiciones de validación y producción de conocimiento que toman un fuerte distanciamiento de las lógicas con las que se produce en las "disciplinas puras y duras", y que por mucho tiempo direccionó los procesos de formación de profesores bajo la premisa de una epistemología positivista.

Pensar el conocimiento didáctico como un referente epistemológico del conocimiento profesional docente, es reconocer en la didáctica una disciplina que emerge y se sustenta en una cultura académica, cuya estructura da cuenta de las investigaciones y de unas luchas internas y externas conducentes a posicionar el conocimiento, así como el reconocimiento de un capital simbólico, los mecanismos de restricción de ingreso al campo y la exclusión de los que no dan cuenta de cierto nivel argumentativo al respecto, o se distancian de las lógicas de la respectiva comunidad académica (Bourdieu, 2003).

Asimismo, es importante aclarar que las investigaciones en torno al conocimiento profesional del profesor, son pensadas desde diversas posturas paradigmáticas y por ende las epistemologías que emergen allí se nutren de dichos paradigmas. De ahí que se pueda decir que el objeto de la didáctica como disciplina afincada en un conocimiento didáctico, no solo puede llegar a ser multiparadigmática (Shulman, 1997), sino que por sí misma es un paradigma que configura modelos de pensar y actuar del profesor y se constituye en un referente para la reflexión y la investigación del fenómeno de la enseñanza. En otras palabras, tiene dos funciones: provee de categorías de análisis al investigador del conocimiento del profesor, a la vez que "dota" al profesor de referentes que le permiten potenciar sus prácticas de la enseñanza, desde la investigación, el análisis y la reflexión.

Ahora bien, vislumbrado un panorama general de la didáctica como disciplina y de cuyo conocimiento didáctico pueden emerger categorías que se constituyen en referentes epistemológicos se estructura el presente ejercicio de ensayo en torno a dos preguntas orientadoras: ¿Qué desafíos implica para el énfasis de investigación sobre el conocimiento profesional del profesor, un referente epistemológico desde el conocimiento didáctico?, ¿En qué medida el conocimiento didáctico -que es el mismo de la disciplina didáctica- puede 
Bio - grafia. Escritos sobre la Biología y su Enseñanza. ISSN 2027

Edición Extraordinaria. p.p. $905-916$

Memorias del VIII Encuentro Nacional de Experiencias en Enseñanza de la Biología y la Educación Ambiental. III Congreso Nacional de Investigación en Enseñanza de la Biología.

constituirse en el referente epistemológico principal del conocimiento profesional del profesor? A continuación se abordan dichas preguntas desde unos marcos generales.

\section{Tendencias de investigación en el énfasis sobre el conocimiento del profesor}

Las investigaciones asociadas con el pensamiento del profesor, se focalizan mayoritariamente en la "...descripción y comprensión de los procesos y constructos que direccionan su conducta- toma de decisiones de aula..." (Álvarez y Moreno, 2012, p. 27); las diversas concepciones y obstáculos epistemológicos que subyacen la naturaleza de sus prácticas (Porlán, Rivero y Pozo 1997), así como ahondar en una explicación lógica del cómo y porqué sus actividades profesionales evidencian ciertos roles y características que emergen de un proceso de formación profesional, se complejizan en el terreno de la práctica y se vinculan con el asunto de valores idiosincráticos. Así, investigar el pensamiento del profesor demanda un panorama complejo y la revisión de una diversidad epistemológica tendiente a la comprensión de sus prácticas pedagógicas, lo cual supone un ejercicio de argumentación y posicionamiento por parte de quien investiga. (Perafán, 2004).

En este orden, son varias las tendencias que movilizan la investigación desde distintas perspectivas y posturas epistemológicas, muchas de ellas prescindiendo del "capital simbólico" de la disciplina de la didáctica como sustrato del conocimiento profesional del profesor, o implícitamente tomando posibles discursos teóricos del conocimiento didáctico sin el respectivo reconocimiento de esta como territorio de saber, o por el contrario respondiendo a otra multiplicidad de lógicas. Aun así, es claro que las diversas miradas al conocimiento del profesor contribuyen al asentamiento de las bases para reconocer en el profesor un conocimiento que lo valida en su ejercicio y los posiciona como un profesional de una comunidad académica con linderos. Roa (2015) haciendo seguimiento en este sentido, ha identificado las siguientes tendencias (ver tabla1)

Tabla 1. Identificación de tendencias de investigación sobre el pensamiento-conocimiento del profesor de ciencias

\begin{tabular}{|l||l|l|}
\hline \multirow{2}{*}{ Tendencias } & \multicolumn{2}{|c|}{ Perspectiva/Categoría } \\
\cline { 2 - 3 } & Roa (2012) & $\begin{array}{c}\text { Lederman y } \\
\text { Lederman (2015) }\end{array}$ \\
\hline
\end{tabular}


Bio - grafia. Escritos sobre la Biología y su Enseñanza. ISSN 2027

Edición Extraordinaria. p.p. $905-916$

Memorias del VIII Encuentro Nacional de Experiencias en Enseñanza de la Biología y la Educación Ambiental. III Congreso Nacional de Investigación en Enseñanza de la Biología.

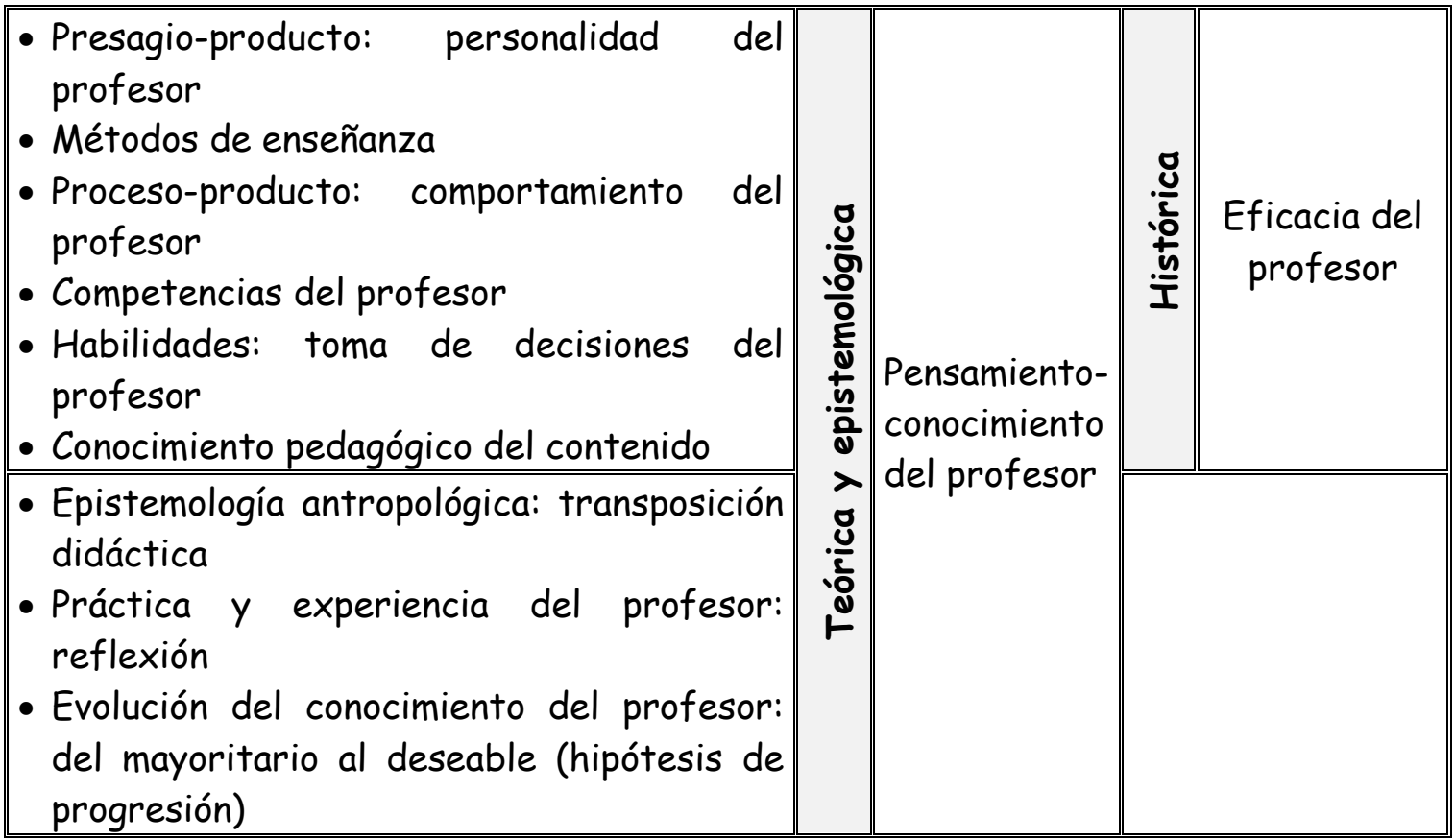

La pluralidad en dichas tendencias concebidas en perspectivas teóricas y epistemológicas (Roa, 2012) o históricas (Lederman y Lederman, 2015), es precisamente lo que enriquece el énfasis de investigación, pero que aun así los distintos grupos de investigación tendientes a caracterizar el conocimiento "profesional" del profesor precisan de miradas distintas y posicionamientos que dan cuenta de la perspectiva en la que se encuentran. Asimismo, es claro que las tendencias de investigación se nutren de posturas epistemológicas, ontológicas $e$ incluso metodológicas que se circunscriben en un paradigma o dentro de la diversidad paradigmática. Cada énfasis precisa de una tendencia en un paradigma, y es apenas natural que así suceda, sin embargo, el arraigamiento y ciertos fijismos epistemológicos e incluso metodológicos a la hora de "caracterizar dicho conocimiento", está generando perspectivas obsoletas y un "sinsabor investigativo" que lejos de profundizar y ampliar el espectro de comprensión del fenómeno o suceso, está generando miradas reduccionistas sobre el conocimiento del profesor. Es evidente, que dichos "arraigos y fijismos epistemológicos" en determinadas tendencias y perspectivas, lejos de reconocer un "conocimiento profesional del profesor" desde la dimensión de la integridad de diversos componentes, puede incurrir notoriamente en posturas exclusivas y excluyentes que hegemonizan la investigación desde una sola mirada y por tanto, aseverar 
Bio - grafia. Escritos sobre la Biología y su Enseñanza. ISSN 2027

Edición Extraordinaria. p.p. $905-916$

Memorias del VIII Encuentro Nacional de Experiencias en Enseñanza de la Biología y la Educación Ambiental. III Congreso Nacional de Investigación en Enseñanza de la Biología.

clasificaciones de los maestros netamente desde su saber disciplinar, sus intrincadas concepciones y creencias, su afectividad y su colectivo cultural e histórico, entre otras, son estilos particulares de abordar un solo foco del asunto en cuestión, y de no existir la respectiva "vigilancia epistemológica" a dicho conocimiento, podría evocar resultados investigativos donde se incurren en juicios que más allá de ser reflexionados son etiquetas derivadas de taxonomías o categorías previamente fijadas. Esto se ilustra cuando se clasifican a los profesores como "...realistas, ingenuos, empiristas, racionalistas..." (Perafán, 2004, p.75), "...o espontaneista, tradicional, instruccional, integrador-transformador..." Martínez (citado por Martínez, Valbuena, Molina y Hederich, 2013, p.33), autoeficaces, intuitivos, prácticos, entre otros. El problema no estriba en la categoría por sí misma, sino en el posicionamiento del investigador, quien asume la posibilidad de atribuirle nuevas significaciones, o de hacer emerger nuevas categorías, o por el contrario ser asumidas como prescripciones epistemológicas y paradigmáticas que mutilan la entidad a ser explorada. De ahí la necesidad de la apertura a diversas posibilidades y no la restricción previa.

En este sentido, y en clave de los enunciados de Kuhn (2004) sobre el comportamiento del conocimiento, se puede expresar que es probable que muchos de estos paradigmas y las consecuentes limitaciones epistemológicas que se proveen, estén en crisis y que por tanto la acumulación de ciertas "anomalías" dentro del desarrollo de la "ciencia normal" pueda estar gestando revoluciones. Esto podría ser el caso de las tendencias -ver tabla 1- sobre la epistemología antropológica (Chevallard, 1991); conocimiento pedagógico del contenido (Shulman, 1986; Shulman, 1987); la evolución del conocimiento del profesor: del mayoritario al deseable (hipótesis de progresión) (Porlán y Rivero, 1998).

Son precisamente, dichas crisis las que ponen en decadencia ciertas tendencias investigativas en el énfasis sobre el conocimiento del profesor. Así pues, las tendencias que se han logrado mantener por cierto tiempo son proclives a debilitarse ante el surgimiento de nuevas problemáticas y desafíos que no pueden ser resueltos con los marcos existentes.

Por otra parte, es de dudosa existencia la premisa de fortalecer una comunidad académica que sugiere la "igualdad" en términos de las producciones teóricas-discursivas y sus precisiones epistemológicas, situaciones como éstas, se distancian de un verdadero territorio de conocimiento, que se sabe, avanza debido a las continuas pujas y 
Bio - grafia. Escritos sobre la Biología y su Enseñanza. ISSN 2027

Edición Extraordinaria. p.p. $905-916$

Memorias del VIII Encuentro Nacional de Experiencias en Enseñanza de la Biología y la Educación Ambiental. III Congreso Nacional de Investigación en Enseñanza de la Biología.

confrontaciones académicas. Es la sensación de unas teorías desplazando otras por la calidad de sus argumentaciones y los marcos propuestos para abordar el fenómeno, es un desplazamiento que se produce como efecto "natural" de los niveles de rigurosidad en las investigaciones adelantadas y las confrontaciones académicas.

El conocimiento didáctico y su devenir epistemológico en el conocimiento profesional del profesor. Algunas implicaciones y desafíos.

Ahora bien, abrir un panorama general desde la perspectiva kuhniana de las revoluciones científicas, las cuestiones epistemológicas y la teoría de campo de Bourdieu (2003), dan cuenta de la especificidad de lo que se intenta abordar,- conocimiento didáctico y su devenir epistemológico en el conocimiento profesional del profesor-, toda vez que aportan a la comprensión de lo que puede suceder y "sucede" en las comunidades académicas de los profesores si están posicionados desde la disciplina de la didáctica; asimismo, el intento de "...establecer una distinción entre los aspectos sociales de las sociedades de conocimiento y las propiedades epistemológicas de las formas de conocimiento, además de examinar con atención como ambas se influyen recíprocamente." (Becher, 2001, p. 16).

En asunción a esto último, es claro que referir la didáctica como disciplina de conocimiento, lejos de discusiones que buscan desconocerla, le han abonado "estatus" epistemológico con sus posturas y la dinámica que se consigue con la investigación. Hacer rastreo a las propiedades epistemológicas de la didáctica, así como su vínculo con los diversos aspectos sociales y su posible posicionamiento epistemológico como fundamento del conocimiento profesional del profesor se denota relevante.

Sin pretender profundizar, cabe decir que el hecho que la didáctica establezca un territorio académico propio no niega su dependencia con el territorio soberanía de los estados, lo que se busca es que existan niveles de autonomía suficientes como para que el campo se desarrolle bajo sus propios objetos: enseñanza, aprendizaje, currículo, entre otros. Si bien es cierto que el campo de la didáctica y pedagogía tienen mucho que ver con lo social, este no es su objeto como lo han querido hacer ver las ciencias sociales o humanas, tampoco sus referentes epistemológicos se encuentran afincados en las ciencias naturales, puras y duras (Roa, 2015).

Ahora, asumiendo posturas que sustentan la idea de la didáctica como disciplina (Díaz Barriga, 1995; Gil, Carrascoa y Martínez,1999; Izquierdo y Estany,2001; Zambrano, 2005; Camilloni, 2008; Roa, 2015), es evidente que la discusión en términos de su configuración 
Bio - grafia. Escritos sobre la Biología y su Enseñanza. ISSN 2027

Edición Extraordinaria. p.p. $905-916$

Memorias del VIII Encuentro Nacional de Experiencias en Enseñanza de la Biología y la Educación Ambiental. III Congreso Nacional de Investigación en Enseñanza de la Biología.

como cuerpo teórico, y por tanto como un campo específico de conocimientos que toma asidero en la investigación sobre el fenómeno de la enseñanza, es una discusión del "pasado", son varias las justificaciones de la didáctica y la trayectoria histórica que le han sustentado su estatus de disciplina.

Pero aun así, llama la atención que pese a los trabajos de "...revisión e historización de antecedentes metodológicos para llegar a la génesis de la didáctica en la obra de Comenio, por ser el pensador históricamente más representativo en el tema y porque a partir del mismo, es posible focalizar la mirada en lo que la didáctica fue en sus primeras gestas..." (Civarolo,2008,p.22), es a partir de allí donde la idea de didáctica produce mutaciones o quizás efecto de la crisis del carácter instrumental y prescriptivo, muchas veces en contra de la psicología del niño, afronta estadios metamórficos que la han desplazado del carácter sustantivo a lo adjetivo, y cuya connotación de disciplina es tan poco aprehendida por la mayoría de los profesores en ejercicio, que referir términos como didáctica, pedagogía y educación se confunden y se presentan de manera indistinta, como una masa poliforma que brinda un rasgo distintivo al conocimiento profesional del profesor.

Algún tipo de conocimiento, con un rasgo particular ha de dar sustento epistemológico a la profesión, y en efecto lo son las disciplinas de la didáctica y la pedagogía, pero no obstante, el panorama histórico tan difuso y la escuela permeada por todo tipo de influencias sociales, culturales y políticas, a lo que suma un nivel formativo deficiente en pregrado de dichas disciplinas, las concepciones y prejuicios distorsionados de la didáctica, así como la misma psicologización de la didáctica y su posterior transformación en tecnología educativa; el desplazamiento de parte de su territorio al currículo, a las "ciencias de la educación" y su conocimiento desdibujado en muchas ocasiones por la opacidad histórica, y su consecuente deslegitimación y vulgarización por cuantas profesiones quisiesen opinar al respecto, generó una confusión reinante en torno al tema y por tanto un posible retroceso en la configuración de la didáctica como disciplina que sustenta la formación docente y su consecuente práctica profesional.

Desde esta perspectiva, sustentar la formación y el conocimiento profesional del profesor, es un asunto que va más allá de comprenderlo psicológicamente y en una suerte de subterfugio, reducirlo a la somera búsqueda de la comprensión y atribución de sentido a las prácticas de la enseñanza. No se trata de buscar "sentidos o atribuir sentidos ciegos a las prácticas" y mucho menos de caer en el positivismo y sus sesgos epistemológicos o la mente en la cuba cartesiana. Se trata de la posibilidad de un posicionamiento de la didáctica y la 
Bio - grafia. Escritos sobre la Biología y su Enseñanza. ISSN 2027

Edición Extraordinaria. p.p. $905-916$

Memorias del VIII Encuentro Nacional de Experiencias en Enseñanza de la Biología y la Educación Ambiental. III Congreso Nacional de Investigación en Enseñanza de la Biología.

pedagogía como disciplinas distintivas del conocimiento profesional, con implicaciones en los programas y planes de formación pregradual que hagan de dichas disciplinas un saber integrado y no yuxtapuesto como se ha presentado. La didáctica genera más compromiso ético con nuestra comunidad académica, precisamente porque al su interior se generan "...relaciones intrincadas y complejas entre las didácticas generales y específicas..." (Camilloni, 2008, p. 26), la idea de especificidad en las didácticas genera un alto grado de complejidad aún mucho mayor, en tanto que requiere de profesionales que se formen en esas gramáticas particulares.

En ese orden, las discusiones epistemológicas y metodológicas alrededor de la enseñanza, son disertaciones que solo pueden ser posibles dado el capital simbólico de la didáctica. Sin lugar a deslegitimar la pedagogía y su papel en la reflexión de la "educación y la enseñanza como prácticas sociales", la pedagogía no tendría mucho que aportar a dicha discusión, precisamente porque su carácter es más ideológico y filosófico, y su "...saber se humaniza..." Foucault (citado por Quiceno,2004), y por supuesto que el saber debe humanizarse, de ninguna manera se desvincula del sujeto, y es precisamente ese valor humano y subjetivo el que crea relaciones de complementariedad con la didáctica, de ahí que no se pueda referir didáctica sin pensar en la pedagogía.

Ahora bien, se han vislumbrado algunas de las implicaciones que tiene para el énfasis de investigación sobre el conocimiento del profesor, un referente epistemológico desde el conocimiento didáctico. Por un lado, el fuerte panorama difuso que presentó históricamente y su consecuente configuración epistemológica, hecho que la distanció por un gran lapso de tiempo como referente de análisis del conocimiento profesional del profesor; su reduccionismo a la política educativa; la instrumentalización de la misma y la supremacía de las ciencias puras y duras en la formación docente, en detrimento de la disciplina de la didáctica, así como un sinnúmero de tesis pregraduales que distaban de un nivel de apropiación al respecto.

Estos a su vez fueron algunos de los problemas a los que se ha venido enfrentando la didáctica, pero que precisamente hacen renacer la didáctica con unos desafíos particulares en el contexto de la formación docente, la actualización de las prácticas de enseñanza y el posicionamiento de la didáctica como disciplina. Estimar los desafíos que la didáctica supone como sustento epistémico de las investigaciones en el conocimiento profesional, acarrea implicaciones en las necesidades formativas de los profesores y una revisión rigurosa a las prácticas de la enseñanza que develen el despliegue de actitudes, toma de 
Bio - grafia. Escritos sobre la Biología y su Enseñanza. ISSN 2027

Edición Extraordinaria. p.p. $905-916$

Memorias del VIII Encuentro Nacional de Experiencias en Enseñanza de la Biología y la Educación Ambiental. III Congreso Nacional de Investigación en Enseñanza de la Biología.

decisiones, incursión de estrategias e incluso discursos que beben de la didáctica y la pedagogía como disciplinas fundantes de su saber, y que en consecuencia configuren el conocimiento profesional en términos de la comunidad académica y lo que acarrea pertenecer a ella, a saber la investigación, la confrontación e incluso las crisis, luchas y tensiones en la apropiación y creación de un capital simbólico.

\section{Bibliografía}

Álvarez, L, y Moreno, M. (2012). El pensamiento del profesor: entre la teoría y la práctica. Bogotá DC. Universidad Nacional de Colombia.

Becher, T. (2001). Tribus y territorios académicos. La indagación intelectual y las culturas de las disciplinas. Barcelona: Gedisa.

Bourdieu, P. (2003). El oficio del científico. Ciencia de la ciencia y la reflexividad. Barcelona Anagrama.

Camilloni, A; Cols, E; Basabe, L; y Feeney, S. (2008). El saber didáctico. Buenos Aires: Paidós.

Chevallard, Y. (1991). La Transposición Didáctica. Del Saber Sabio al Saber Enseñado. Buenos Aires: Aique.

Civarolo, M. (2008).La idea de didáctica. Antecedentes, génesis y mutaciones. Bogotá: Cooperativa editorial magisterio.

Diaz Barriga. (1995). Didáctica. Aportes para una polémica. Argentina: Aique.

Estany, A., Izquierdo, M. (2001). Didactología: una ciencia de diseño. Éndoxa, 14,13-33.

Gil-Pérez, D., Carrascosa, J; y Martínez F. (1999). El surgimiento de la didáctica de las ciencias como campo específico de conocimientos. Revista de Educación y Pedagogía, 11 (25), $13-65$.

Kuhn, T. (2004) La estructura de las revoluciones científicas. México: Fondo de cultura económica. 
Bio - grafia. Escritos sobre la Biología y su Enseñanza. ISSN 2027

Edición Extraordinaria. p.p. $905-916$

Memorias del VIII Encuentro Nacional de Experiencias en Enseñanza de la Biología y la Educación Ambiental. III Congreso Nacional de Investigación en Enseñanza de la Biología.

Lederman, Ny Lederman, J. (2015). The Status of Preservice Science Teacher Education: A Global Perspective. Journal of Science Teacher Education, 26:1-6.

Martínez, C.,Molina, A., Valbuena, E., y Hederich, C.(2013). El conocimiento profesional de los profesores de ciencias sobre el conocimiento escolar. Resultados de investigación. Bogotá: Cade.

Perafán, G, (2004). La epistemología del profesor sobre su propio conocimiento profesional. Bogotá Historia con futuro.

Porlán, R y Rivero, A. (1998). El conocimiento de los profesores. España: Editorial Díada.

Porlán, R., Rivero, G., Pozo, M (1997). Conocimiento profesional y epistemología de los profesores I: teoría, métodos e instrumentos. Enseñanza de las ciencia, 15 (2), 155-171.

Quiceno, H. (2005). Michael Foucault ipedagogo? En Zuluaga, O; Noguera, C; Quiceno, H; Saldarriaga, O; Sáenz, J; Martínez, A; et al. Foucault, la pedagogía y la educación, pensar de otro modo. Bogotá: Cooperativa Editorial Magisterio.

Roa, A. (2015). Constitución y contenido de la didáctica y pedagogía como disciplinas de conocimiento. Capítulo IV, tomado del documento parcial de la tesis de doctorado: "Configuración del conocimiento profesional didáctico del profesor de ciencias para la enseñanza de la biotecnología en Colombia" Universidad Pedagógica Nacional.

Shulman, L. (1986). Those who understand: Knowledge growth in teaching. Educational Researcher. 15(2), 4-14.

Shulman, L. (1997). Paradigmas y programas de investigación en el estudio de la enseñanza. Una perspectiva contemporánea. En: Wittrock, M. (Editor). La investigación de la enseñanza I. Enfoques, teorías y métodos. España: Ediciones Paidós Ibérica. pp: 9-91.

Shulman, L. S. (1987). Knowledge and teaching: Foundations of the new reform. Harvard Educational Review, 57, 1-22 
Bio - grafia. Escritos sobre la Biología y su Enseñanza. ISSN 2027

Edición Extraordinaria. p.p. 905- 916

Memorias del VIII Encuentro Nacional de Experiencias en Enseñanza de la Biología y la Educación Ambiental. III Congreso Nacional de Investigación en Enseñanza de la Biología.

Zambrano, A.(2005). Didáctica, pedagogía y saber. Bogotá, Colombia: Cooperativa editorial Magisterio. 